\title{
Potenzproblem? Auch an Schlafapnoe denken!
}

Hintergrund und Fragestellung: Patienten mit obstruktiver Schlafapnoe (OSA) leiden in ca. zwei Dritteln der Fälle an einer mehr oder weniger starken erektilen Dysfunktion (ED) [1]. Die OSA scheint ein unabhängiger Risikofaktor für ED zu sein. Eine CPAP-(Continuous Positive Airway Pressure)Therapie reduziert die Häufigkeit von ED-Symptomen bei OSA-Patienten [2]. Ziel der Studie war es, einmal umgekehrt zu untersuchen, wie hoch die Prävalenz von Schlafstörungen, besonders der obstruktiven Schlafapnoe (OSA), bei Patienten mit neu diagnostizierter ED ist.

\section{Patienten und Methoden: In} einer spezialisierten andrologisch-urologischen Ambulanz konnten 129 Patienten mit neu diagnostizierter ED (dies ent-

\section{Originalie}

Kalejaiye O, Abdel Raheem A, Moubasher $A$ et al. Sleep disorders in patients with erectile dysfunction. BJU Int 2017 Jul 15. doi: 10.1111/bju.13961.

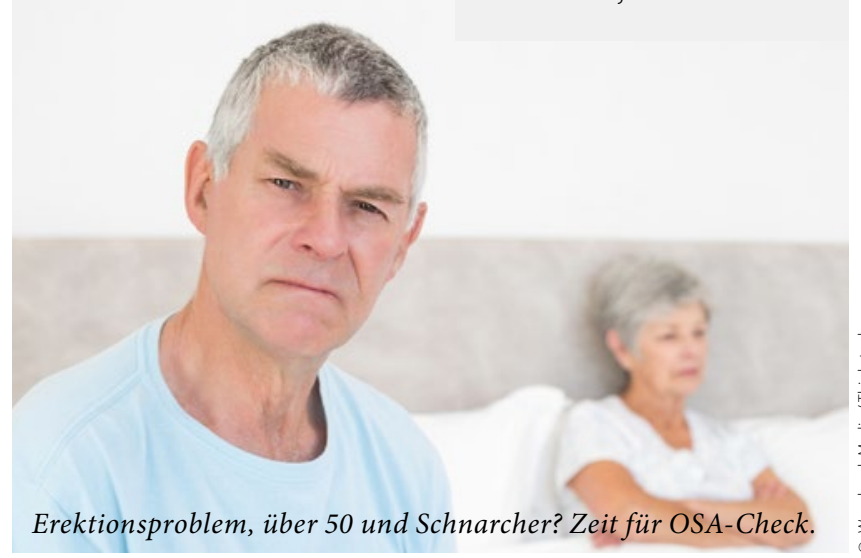

sprach $78 \%$ der in einem Zeitraum von 8 Monaten konsekutiv und prospektiv untersuchten Patienten) mittels validierter Fragebögen auf Schlafstörungen hin untersucht werden, der Fokus lag auf der OSA. Es wurden 3 Fragebögen benutzt: Einer zum Screening auf ED, der „International Index of Erectile Function (IIEF), einer auf OSA sowie der „insomnia severity index“-Fragebogen zur Erfassung anderer Schlafstörungen. Alle 129 Patienten füllten alle drei Fragebögen aus. Ein dringender OSA-Verdacht wurde geäußert, wenn mindestens drei der folgenden acht Kriterien zutrafen:

- Lautes Schnarchen

- Epworth Sleepiness Scale (ESS-)Score > 11

- Fremdanamnestisch beobachtete Atemstillstände

- Arterielle Hypertonie

- Body Mass Index > $35 \mathrm{~kg} / \mathrm{m}^{2}$

- Alter $>50$ Jahre

- Halsumfang $>40 \mathrm{~cm}$

- Männliches Geschlecht

Ergebnisse: Der dringende Verdacht auf OSA ( $\geq 3$ von 8 Items positiv) wurde bei 77 Patienten (55\%) gestellt. Diese Patienten waren signifikant älter $(61,4$ vs. 46,5 Jahre; $\mathrm{p}<0,001)$ und hatten einen signifikant höheren Body Mass Index (29,4 vs. 26,7 kg/ $\left.\mathrm{m}^{2} ; \mathrm{p}<0,001\right)$ als der Durchschnitt des Kollektivs. Die OSAGruppe wies einen signifikant höheren IIEF-ED-Score (6,2 vs. $9,1 ; p=0,018)$ und ebenso einen (nicht signifikant) höheren Insomnie-Schweregrad-Score (7,9 vs. 5,5.; $\mathrm{p}=0,061)$ auf.

Schlussfolgerungen: Männer mit ED haben ein deutlich erhöhtes Risiko, eine nicht diagnostizierte OSA zu haben. Sie sollten daher auch bzgl. einer OSA befragt und ggf. untersucht werden.

\section{- Kommentar von Prof. Dr. med. Kurt Rasche}

\section{Trotz Limitationen ist die Studie ein Weckruf}

Die Arbeit befasst sich mit einem sehr interessanten, klinisch relevanten, aber oft verschwiegenen Thema. Zwischen ED und OSA kann ein unmittelbarer kausaler Zusammenhang diskutiert werden. Intermittierende Hypoxämie und Weckreaktionen führen zu oxidativem Stress sowie nicht erholsamem Schlaf, beides Faktoren, die sowohl zu vaskulärer Reaktion als auch zu psychologischen Veränderungen wie depressiver Verstimmung, Abgeschlagenheit und verminderter Libido führen können. Dieser Studie zufolge leiden offenbar mehr als die Hälfte der Patienten mit ED an einer OSA. Dies erscheint hoch und könnte an dem sehr sensitiven, wahrscheinlich aber nicht so spezifischen OSA-Screening-Fragebogen liegen, was auch die wesentliche Limitation dieser Studie ist.

Dennoch, die Studie ist ein "Weckruf" gerade für Andrologen, Urologen, Psychologen und Psychiater, bei denen sich immer wieder Patienten mit ED einfinden. Die Befragung nach typischen OSA-Symptomen und ggf. die weitere schlaf- medizinische Abklärung des OSA-Verdachts sollte zum diagnostischen Routine-Repertoire bei der Erstdiagnose einer ED gehören!
Literatur
1. Budweiser S et al. J Sex Med 2009;6:3147-57
2. Husnu T el al. African Health Sciences 2015;15:171-9

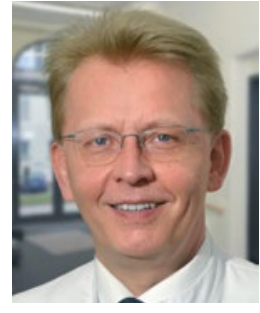

Prof. Dr. med. Kurt Rasche

HELIOS Universitätsklinikum Wuppertal Universität Witten/Herdecke Bergisches Lungenzentrum Heusnerstr. 40, 42283 Wuppertal kurt.rasche@helios-kliniken.de 